University of Wollongong

Research Online

Faculty of Engineering and Information

Faculty of Engineering and Information

Sciences - Papers: Part A

Sciences

January 2006

\title{
Simulation with Geant4 of a novel position detector based on nanotechnologies
}

R Angelucci

Onsiglio Nazionale Delle Ricerche- Imm, Sezione Di Bologna,

M Cuffiani

Universita di Bologna

G M. Dallavalle

Istituto Nazionale di Fisica Nucleare Italy

S Guatelli

University of Wollongong, susanna@uow.edu.au

A Jagminas

Institute Of Chemistry

See next page for additional authors

Follow this and additional works at: https://ro.uow.edu.au/eispapers

Research Online is the open access institutional repository for the University of Wollongong. For further information contact the UOW Library: research-pubs@uow.edu.au 


\title{
Simulation with Geant4 of a novel position detector based on nanotechnologies
}

\begin{abstract}
The application of nanotechnologies for a novel type of position particle detector is the aim of NanoChanT project. Titanium dioxide (TiO2) nanowires are grown in an highly ordered array of parallel nanochannels in an alumina template. The template is obtained from the controlled anodization of an aluminum foil that produces regular pores. Depending on anodization conditions, the diameter of the pores ranges from 20 to $200 \mathrm{~nm}$ with 40 to $500 \mathrm{~nm}$ pitch. After doping, a pn junction can be obtained from each of the TiO2 nanowire. The junction is inversely polarized and is sensitive to the passage of charged particles. The charge produced in groups of nanowires is collected by metal pixels and readout by CMOS electronics. GEANT4 has been used for the simulation of the interaction of ionizing particles with the detector. The simulation at the nanoscale level is of fundamental importance in the design of the geometry of the device, in order to choose the best compromise between the smallest detectable charge produced in a readout channel and the maximum achievable spatial resolution. The current design and status of the detector and the considerations driven by the simulation with GEANT4 are presented.
\end{abstract}

\section{Keywords}

detector, geant4, position, simulation, nanotechnologies, novel

\section{Publication Details}

Angelucci, R., Cuffiani, M., Dallavalle, G. M., Guatelli, S., Jagminas, A., Malferrari, L., Montanari, A., Odorici, F., Pia, M. G., Rizzoli, R. \& Veronese, G. P. (2006). Simulation with Geant4 of a novel position detector based on nanotechnologies. In B. Phlips (Eds.), IEEE Nuclear Science Symposium Conference Record (pp. 1480-1484). New Jersey, United States: IEEE.

\section{Authors}

R Angelucci, M Cuffiani, G M. Dallavalle, S Guatelli, A Jagminas, L Malferrari, A Montanari, F Odorici, M G. Pia, R Rizzoli, and G P. Veronese 


\title{
Simulation with GEANT4 of a Novel Position Detector Based on Nanotechnologies
}

\author{
R. Angelucci, M. Cuffiani, G.M. Dallavalle, S. Guatelli, A. Jagminas, L. Malferrari, A. Montanari, F. Odorici, \\ M.G. Pia, R. Rizzoli and G.P. Veronese.
}

\begin{abstract}
The application of nanotechnologies for a novel type of position particle detector is the aim of NanoChanT project. Titanium Dioxide $\left(\mathrm{TiO}_{2}\right)$ nanowires are grown in an highly ordered array of parallel nanochannels in an Alumina template. The template is obtained from the controlled anodization of an Aluminum foil that produces regular pores. Depending on anodization conditions, the diameter of the pores ranges from 20 to $200 \mathrm{~nm}$ with 40 to $500 \mathrm{~nm}$ pitch. After doping, a pn junction can be obtained from each of the $\mathrm{TiO}_{2}$ nanowire. The junction is inversely polarized and is sensitive to the passage of charged particles. The charge produced in groups of nanowires is collected by metal pixels and readout by CMOS electronics. GEANT4 has been used for the simulation of the interaction of ionizing particles with the detector. The simulation at the nanoscale level is of fundamental importance in the design of the geometry of the device, in order to choose the best compromise between the smallest detectable charge produced in a readout channel and the maximum achievable spatial resolution. The current design and status of the detector and the considerations driven by the simulation with GEANT4 are presented.
\end{abstract}

Index Terms - NanoChanT, Alumina, Nanochannel, Nanowire, Titanium Dioxide, Detector.

\section{INTRODUCTION}

$\mathbf{N}$ ANOTECHNOLOGIES, the ability to organize structures from the atomic to the molecular scales, are viewed as one of the most significant technological frontier being explored. Their applications are relevant in many fields, such as medicine, new materials, electronic circuits and others. In our application, the NanoChanT project [1], [2], we are investigating the possibility to use some already available nanotechnologies in order to build a position particle detector which can improve resolution by at least one order of magnitude with respect to present detectors. We envisage to readout the detector through standard CMOS electronics embedded in a $\mathrm{Si}$ substrate. The present scale of integration of CMOS electronics limits the readout granularity and the corresponding spatial resolution, but future developments might benefit of the nanometric scale

R. Angelucci and R. Rizzoli are with Consiglio Nazionale delle RicercheIMM, Sezione di Bologna, V. Gobetti 101, 40129 Bologna, ITALY.

M. Cuffiani and G.P. Veronese are with Dipartimento di Fisica, Universitá di Bologna, V. Irnerio 46, 40126 Bologna, ITALY.

G.M. Dallavalle, L. Malferrari, A. Montanari and F. Odorici are with Istituto Nazionale di Fisica Nucleare, Sezione di Bologna, V.le B. Pichat 6/2, 40127 Bologna, ITALY (e-mail: alessandro.montanari@bo.infn.it).

A. Jagminas is with Insitute of Chemistry, A. Goštauto 9, 01108 Vilnius, LITHUANIA.

S. Guatelli and M.G. Pia are with Istituto Nazionale di Fisica Nucleare, Sezione di Genova, V. Dodecanneso 33, 16146 Genova, ITALY. of the active part of the detector. In order to dimension correctly the geometry, it is of fundamental importance to simulate the behaviour of an ionizing particle inside the various parts of the detector. This is a challenging task since, up to now, no Monte Carlo program was used at a nanometric scale. The Geant 4 simulation toolkit [3], [4] was the best candidate to do this job and the first results go in the right direction.

\section{The Detector PrinCIPLE}

Two basic nanotechnologies are used in NanoChanT ("Nano Channel Template"): nanochannels and nanowires. The nanochannels are obtained from the controlled anodization of Aluminum ( $\mathrm{Al}_{2} \mathrm{O}_{3}$, Alumina) and have a highly regular honeycomb-like geometry. The nanowires are made of Titanium Diodixe ( $\mathrm{TiO}_{2}$, an intrinsically n-type semiconductor) and are grown inside the nanochannels. The basic idea is shown in Figure 1. A regular and uniform matrix of $\mathrm{TiO}_{2}$ nanowires are

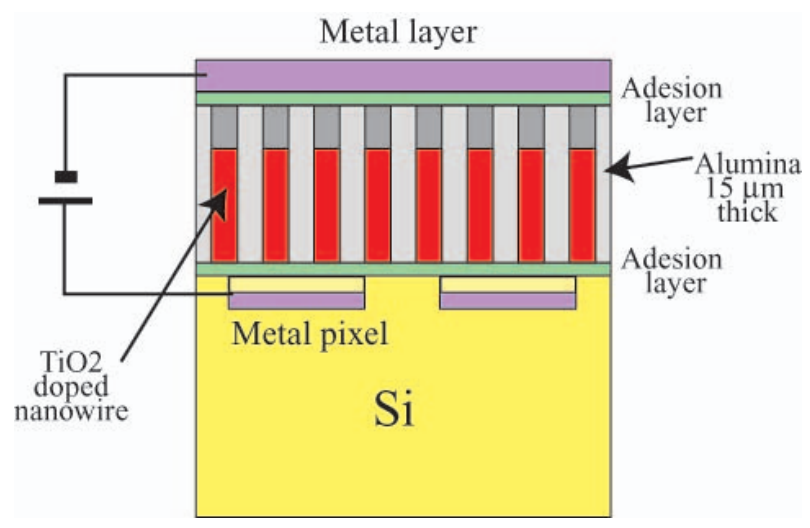

Fig. 1. The basic architecture of NanoChanT detector.

grown inside the Alumina template (with $15 \mu \mathrm{m}$ thickness), which isolates the wires and provides the mechanical stiffness to the object. The nanowires are instrinsically $n$-type semiconductors and are p-doped in order to form p-n junctions that are completely depleted through a reversed bias voltage [6]. Each nanowire constitutes the active part of the detector. When a particle crosses the nanowire, the energy is deposited in the material and electron-hole pairs are produced: the charge is collected through metallic pixels and produces a signal that is proportional to the energy released in the material. The pixels and the CMOS readout electronics are embedded in a silicon substrate, which is attached to the bottom of the 
Alumina template. The nanowires are isolated each other by the Aluminum oxide and act as independent diodes. The charge produced is confined inside the wire and in principle the spatial resolution can scale as the pitch of the matrix. Presently, the scale of integration of CMOS electronics limits the readout granularity to values much larger than nanowires pitch. But, for the future, improvements in the readout electronics could fully benefit of the nanowires geometry. In order to construct such an object, many processing steps were developed and many samples were produced with different conditions. We are presently focusing our activity on the doping of $\mathrm{TiO}_{2}$ nanowires.

\section{A. The Alumina Template}

The basic component for the growth of the ordered array of $\mathrm{TiO}_{2}$ nanowires is the Alumina template. Using a technique similar to the one described in [7], [8], several samples of Alumina templates were produced. The starting point is a high purity Aluminum foil, $100 \mu \mathrm{m}$ thick, which is anodized in an electrolytic cell. Under controlled condition, the current spontaneously creates holes in the Aluminum oxide, with self organization in a regular honeycomb-like structure. The type of acid, the temperature, the voltage bias and the duration of the process are critical parameters through which it is possible to control the size and the pitch of such holes. Typically, by using a 0.3 molar solution of oxalic acid with a voltage bias of $40 \mathrm{~V}$, it is possible to produce nanochannels with a pitch of about $100 \mathrm{~nm}$. By using phosphoric acid with a bias of $190 \mathrm{~V}$, a larger pitch of about $500 \mathrm{~nm}$ can be obtained. The size of the holes diameter scales with the pitch, ranging from about $40 \mathrm{~nm}$, for oxalic acid, to $200 \mathrm{~nm}$, for phosphoric acid. For the success of the process, the preparation of the Aluminum foil is a delicate phase. The foil is degreased and annealed at high temperature in order to remove impurities. Then the surface is treated with an electropolishing process in order to remove defects and improve the planarity. Most important for

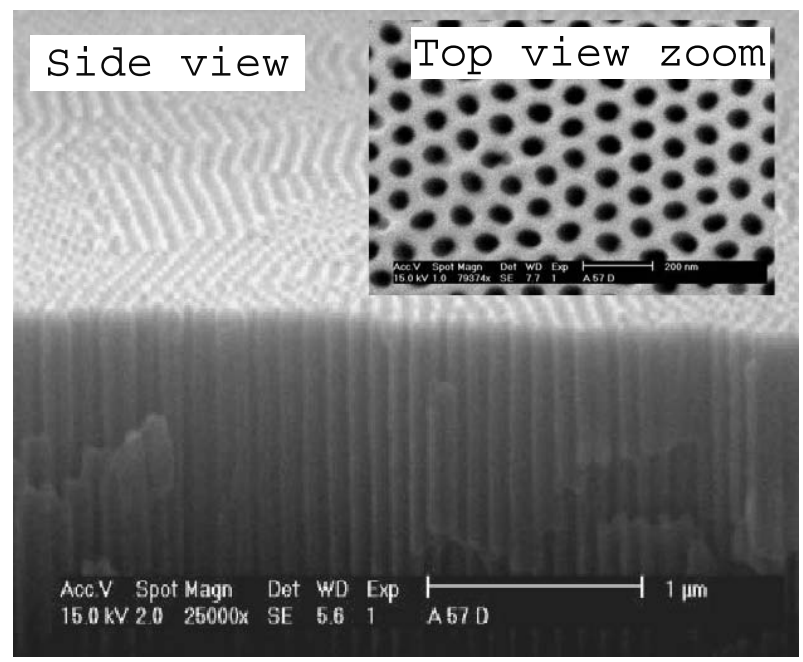

Fig. 2. SEM image of an Alumina sample. our purposes is that Alumina is a ceramic material with a good mechanical strength and is a good electrical insulator. Figure 2 shows a picture taken with a Scanning Electron Microscope (SEM) of an Alumina sample, obtained by using oxalic acid. It represents a side view of the Alumina template. The holes have a diameter of about $40 \mathrm{~nm}$ and a pitch of $100 \mathrm{~nm}$. The regularity of the pattern of the holes is limited by the grain boundaries of the original Aluminum foil. The sample is broken in two halves, in order to show the depth of the straight nanochannels. At a process temperature of about $5^{\circ} \mathrm{C}$, the excavation rate was about $3 \mu \mathrm{m} / \mathrm{h}$, and a full depth of about $100 \mu \mathrm{m}$ was obtained after 30 hours of anodization in the electrolytic cell.

\section{B. Alumina on Silicon}

In order to attach the Silicon substrate to the Alumina template, a dedicated process was developed. The starting point is a Silicon wafer onto which a thin layer of Niobium is sputtered. The Niobium acts as an adhesion layer. A thicker layer of Aluminum can be sputtered on the Niobium surface. Finally the Aluminum is anodized in an electrolytic cell, as described above, in order to form the nanochannels. In Figure 3

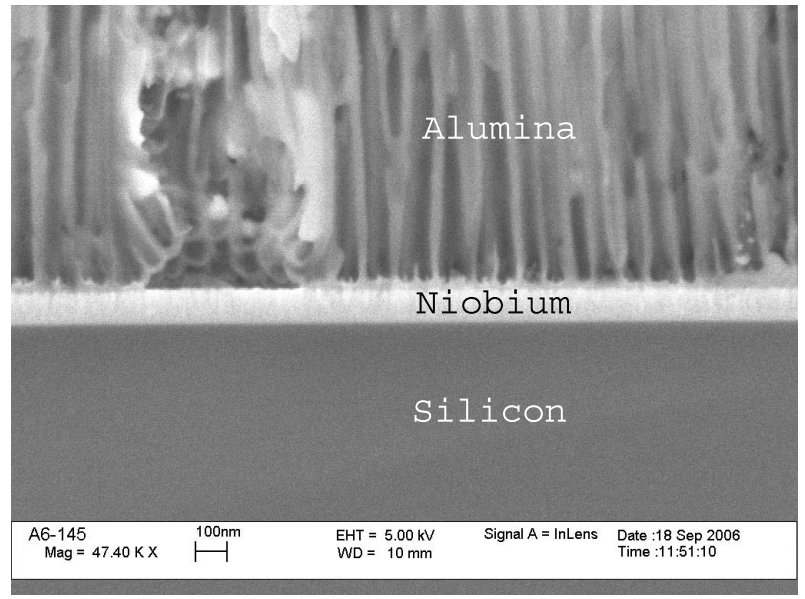

Fig. 3. SEM image of the Alumina template attached to a Silicon layer.

a SEM side image of a sample is shown. The Niobium interface between the Alumina template and the Silicon substrate is clearly visible.

\section{C. $\mathrm{TiO}_{2}$ nanowires}

The deposition of $\mathrm{TiO}_{2}$ inside the nanochannels is performed through an electrolytic reaction in a solution of $\mathrm{TiSO}_{4}$ salts. The process proceeds with a continuos voltage in the range of 2.5-3.5 V and about 5 hours are necessary to deposit $50 \mu \mathrm{m}$ long nanowires. A successive thermal treatment of the sample at about $500^{\circ} \mathrm{C}$ in air is performed to change the phase of $\mathrm{TiO}_{2}$ to crystal (anatase), which is the one with semiconductor properties. In the SEM image in Figure 4, the Alumina template was dissolved with a $\mathrm{NaOH}$ solution in order to make the nanowires visible. 


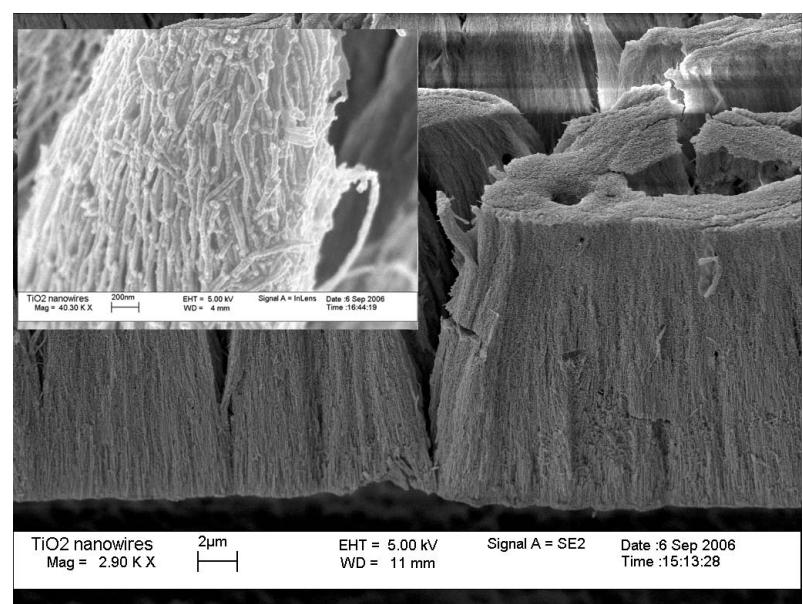

Fig. 4. SEM image of $\mathrm{TiO}_{2}$ nanowires (after Alumina removal).

\section{Simulation with GEANT4}

Geant4 is a toolkit for simulating the passage of particles through matter. It includes a complete range of functionality including tracking, geometry, physics models and hits. The physics processes offered cover a comprehensive range, including electromagnetic, hadronic and optical processes, a large set of long-lived particles, materials and elements, over a wide energy range. The lower limit of the energy range is of particular importance in our case: the lower the limit is on the minimum energy, the better is the description of the physics at small scale (in terms of total energy deposited in a material and its spatial distribution). In particular, the Low Energy Electromagnetic (EM) package (Livermore library) is able to simulate processes down to $250 \mathrm{eV}$ [5], that corresponds to a range of about $300 \mathrm{~nm}$ of an electron in Titanium Dioxide. For this reason, the first studies of application of Geant 4 toolkit to our case were performed by using electrons at the minimum of ionization (corresponding to an electron momentum of $2 \mathrm{MeV} / \mathrm{c}$ ) as primary particle and activating the Low Energy EM package to simulate the relevant physics processes for our application: continuos ionization, production of secondary electrons, multiple scattering.

\section{A. Preparatory test}

In order to understand the behaviour of Geant 4 at sub-micron scales, as a preparatory test, we studied a simple case: $2 \mathrm{MeV}$ electrons incident perpendicularly over a disc made of Titanium Dioxide, $300 \mathrm{~nm}$ thick. The energy released was integrated to get the total energy deposited inside the target. The distribution, obtained by simulating 200k events, is shown in Figure 5 .

The mean energy deposited by the primary electron is about $85 \mathrm{eV}$. The distribution presents a small tail at higher energies around $600 \mathrm{eV}$ : this tail corresponds to the energy released by secondary electrons ( $\delta$ rays) that are generated in about 5\% of the events (see Figure 6). These secondary particles release more energy because they are slower than the primary electron,

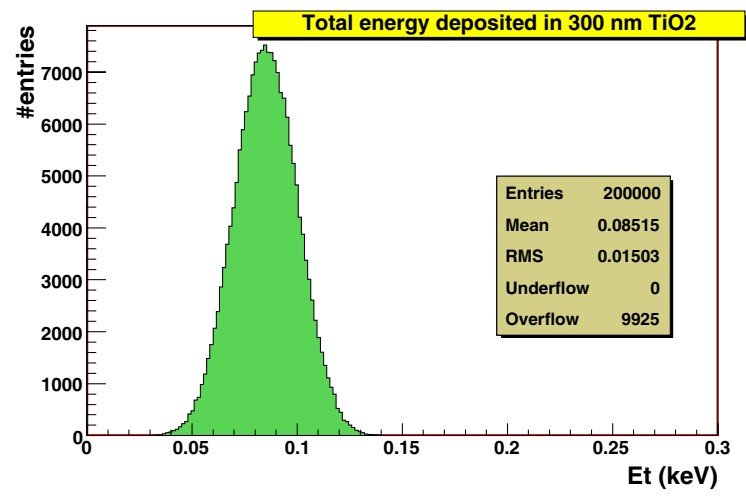

Fig. 5. Total energy deposited in a $\mathrm{TiO}_{2}$ layer ( $300 \mathrm{~nm}$ thick) by a $2 \mathrm{MeV}$ electron.

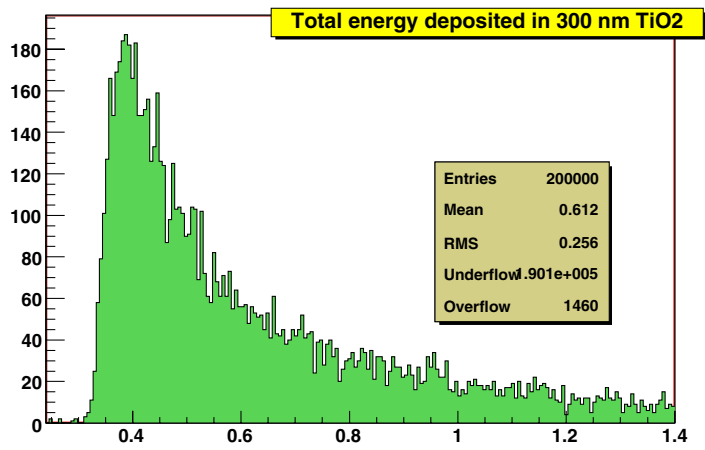

Fig. 6. Tail in total energy deposited, due to $\delta$ rays.

with a momentum spectrum starting at $250 \mathrm{eV}$, that corresponds to the minimum energy cut of the Low Energy EM package.

\section{B. Simulation of $\mathrm{TiO}_{2}$ matrix in Alumina}

Since the preparatory test gave reasonable results, the following step was the setup of a simplified version of the geometry of NanoChanT detector. An Alumina box of $2.5 \mu \mathrm{m} \times 2.5 \mu \mathrm{m} \times 10.0 \mu \mathrm{m}$ was placed in vacuum with the long side along the $\mathrm{z}$ axis. Inside the box, $\mathrm{TiO}_{2}$ cylindrical nanowires were placed with their axis along the $\mathrm{z}$ direction and with an honeycomb-like geometry in the $\mathrm{x}-\mathrm{y}$ plane. As a first test, a diameter of $200 \mathrm{~nm}$ and a pitch of $250 \mathrm{~nm}$ were choosen. A view in the $x-y$ plane is shown in Figure 7. Electrons with $2 \mathrm{MeV} / \mathrm{c}$ momentum were fired on the target, with an angle of 30 degrees with respect to the normal of the $x-y$ plane. The projection of the primary particle direction onto the $x-y$ plane is depicted in Figure 7 (red line). The nanowires that are intersected by this line lay on a diagonal. The graphical output of the simulation of 100 events is shown in Figure 8, in which all the events are superimposed on the same picture. On the right side all generated electrons lay on the same line. After entering the target, they undergo physics interactions with the material and are deviated from the initial direction and in some cases they generate secondary electrons. The total energy 


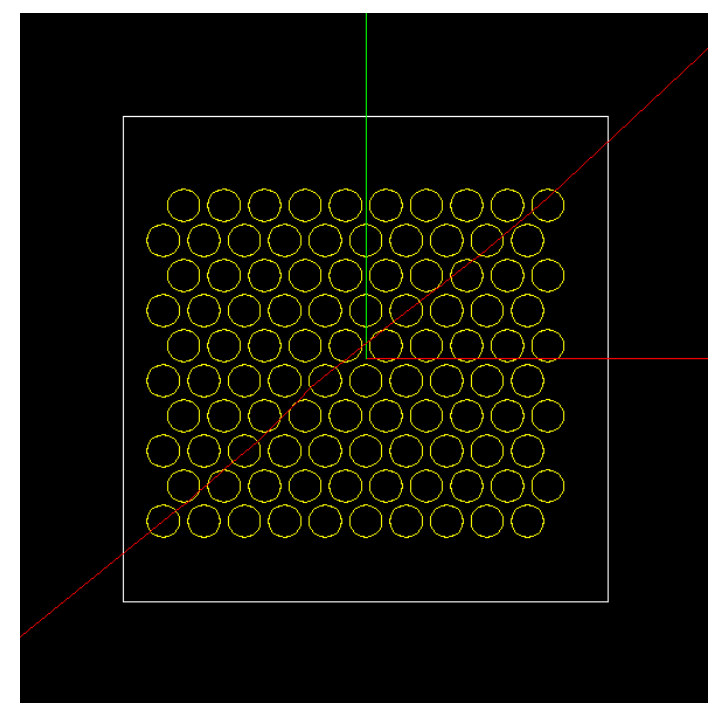

Fig. 7. $x-y$ view of the Alumina box with nanowires inside.

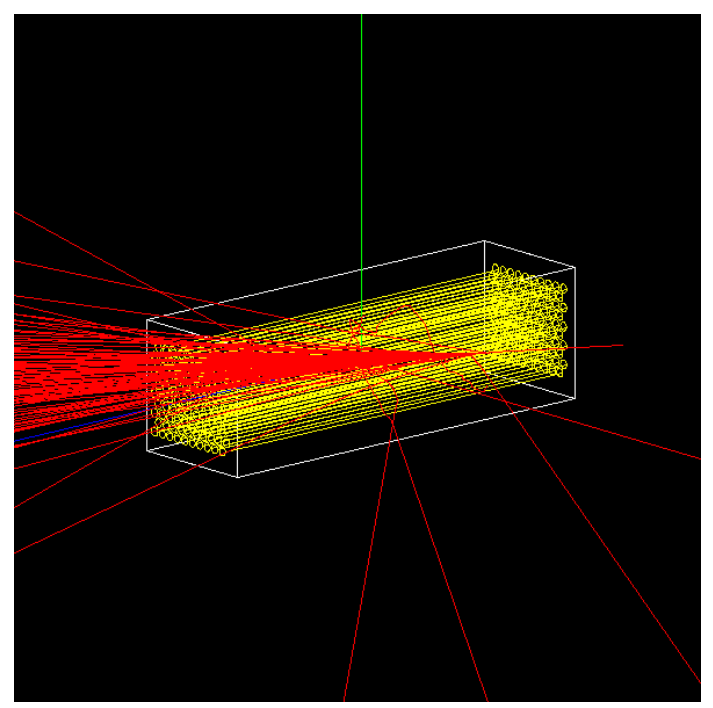

Fig. 8. Graphical output of Geant4 simulation of $2 \mathrm{MeV}$ electrons fired on a matrix of $\mathrm{TiO}_{2}$ nanowires inside the Alumina template (100 events superimposed).

deposited in each nanowire is recorded for each event. The distribution (corresponding to 200k events) of the total energy deposited in a single nanowire is shown in Figure 9: the mean value is about $180 \mathrm{eV}$. It should be noted that in this case the mean is higher than the one observed in the preparatory test, because the material thickness crossed by an inclined track can be much higher than the diameter of the tube $(200 \mathrm{~nm})$. The number of hits for each nanowire was also recorded, where, in this case, we define that a nanowire has an hit if it is touched by the primary particle or its secondaries. The hit map, corresponding to 200k events, is shown in Figure 10. As can be expected, the hits lay along the same diagonal correponding to the projection on the primary particle direction onto the $\mathrm{x}$ y plane. On the bottom-left area, the hits correspond to the

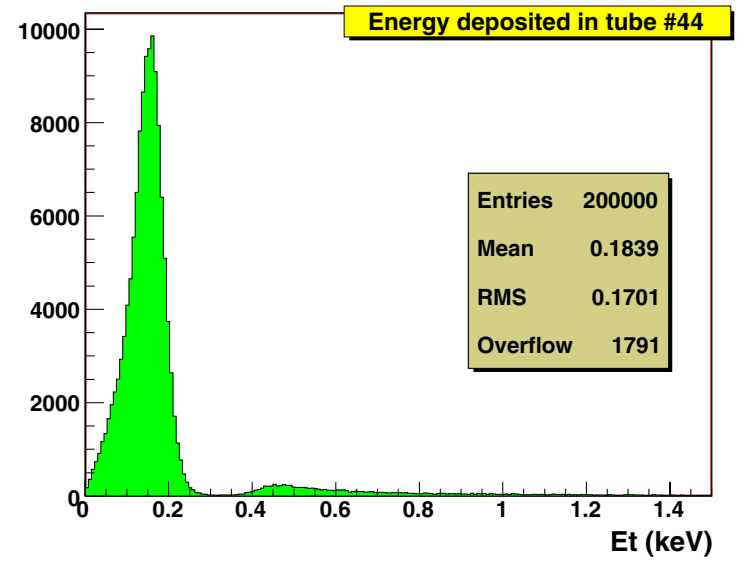

Fig. 9. Total energy deposited in a single nanowire (200k events).

nanowires that are encountered by the primary particle when entering the target. On the top-right area they correspond to the nanowires touched when exiting the target and they are more dispersed because the track has undergone to interactions inside the target. In any case, the dispersion is of negligible amount: this fact suggests that the position resolution will not be affected strongly by charge diffusion inside the detector.

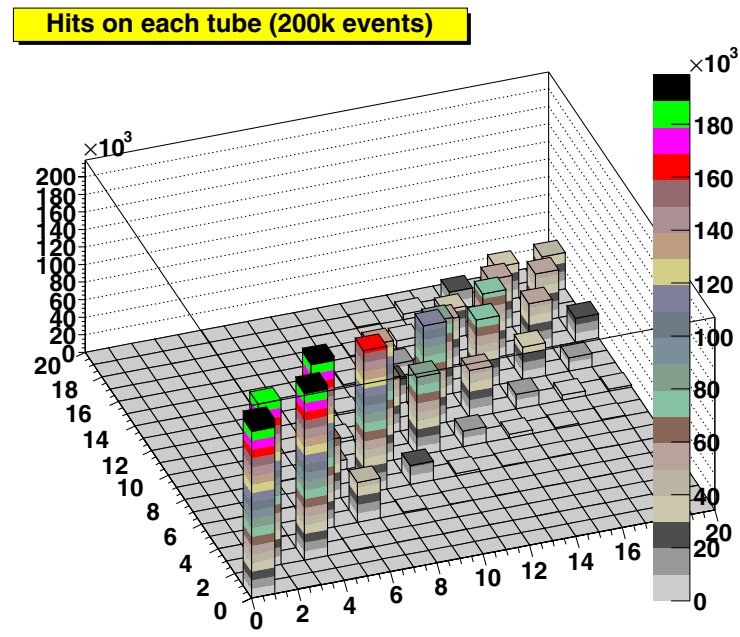

Fig. 10. Occupancy map of the hits on each nanowire (integrated over 200k events).

\section{An hypothesis of readout geometry}

Even if the results of the previous simulations are very preliminary, it is possible to get an estimation of the maximum amount of charge that can be collected, if we readout the nanowires through metallic pixels. In the hypothesis of a pixel size of $1.0 \mu \mathrm{m} \times 1.0 \mu \mathrm{m}$, with the simulated geometry, the charge of about 10 nanowires would be fully collected by one single pixel. Assuming a mean energy deposit of about $150 \mathrm{eV}$ per nanowire, in total about $1500 \mathrm{eV}$ would be deposited in the 
set of nanowires corresponding to one pixel. In $\mathrm{TiO}_{2}$, about $10 \mathrm{eV}$ are necessary to create an electron-hole pair: this means that a maximum of about 150 electrons can be collected by one single pixel. This amount of charge would be a readable signal with the standard CMOS technology.

\section{PERspectives AND CONCLUSION}

From the point of view of the detector development, we are setting up the last step necessary for the assembly of a prototype: that is, the doping of $\mathrm{TiO}_{2}$ nanowires inside the Alumina template. We aim to produce a simplified version of the device in which we do not have pixels, but a unique metallic pad. The signal collected by this pad will be read and amplified with standard electronics. We have setup a test system in which the response of the simplified detector will be tested with radioactive sources. This setup will be simulated with Geant4. This test will allow to validate the Monte Carlo with real data. On the other hand, the cross check of real data with simulation, will allow to determine the efficiency of charge collection, which is a critical aspect in the development of this detector.

In conclusion, the challenge to build a detector based on nanotechnologies is going on and we aim to produce soon a simplified version of the detector. In parallel, the first test to simulate the nanoscale device with Geant4 has been made. In particular, the Low Energy Electromagnetic package seems to be able to reproduce the physics processes at sub-micron scale. These results will be soon cross checked with real data. In general, the simulation of radiation-matter interaction at sub-micron scale could open perspectives also for other applications.

\section{REFERENCES}

[1] R. Angelucci et al., Application of Nanotechnologies in High Energy Physics, Nucl. Phys.B (Proc. Suppl.), 125 (2003) 164-168.

[2] R. Angelucci et al., A novel position detector based on nanotechnologies: the NanoChanT project, Nucl. Phys.B (Proc. Suppl.), 150 (2006) 140-143.

[3] S. Agostinelli et al., GEANT4-a simulation toolkit, Nucl. Instr and Meth. A, 506 (2003) 250-303.

[4] J. Allison et al., GEANT4 Developments and Applications, IEEE Trans. on Nucl. Sci., Vol. 53, N. 1 (2006) 270-278.

[5] S. Chauvie, S. Guatelli, V. Ivanchenko, F. Longo, A. Mantero, B. Mascialino et al., GEANT4 Low Energy Electromagnetic Physics, Conf. Rec. 2004 IEEE Nucl. Sci. Symp., Vol. 3, (2004) 1881-1885.

[6] Y. Chen et al., Preparation of a novel $\mathrm{TiO}_{2}$ based p-n junction nanotube photocatalyst, Env. Sci. and Tech. Vol. 39, 5 (2005).

[7] O. Jessensky et al., Appl. Phys. Lett., 72 (1998) 1173.

[8] H. Masuda et al., Science, 268 (1995) 1466. 